\title{
Les circulations en santé : des produits, des savoirs, des personnes en mouvement : argumentaire
}

Chiarella Mattern et Audrey Bochaton

\section{(2) OpenEdition \\ Journals}

Édition électronique

URL : http://journals.openedition.org/rfst/632

DOI : $10.4000 /$ rfst.632

ISSN : 2492-3672

Éditeur

Espaces et SOciétés (UMR 6590)

Référence électronique

Chiarella Mattern et Audrey Bochaton, «Les circulations en santé : des produits, des savoirs, des personnes en mouvement : argumentaire », Revue francophone sur la santé et les territoires [En ligne] Les circulations en santé : des produits, des savoirs, des personnes en mouvement, mis en ligne le 16 novembre 2019, consulté le 06 avril 2021. URL : http://journals.openedition.org/rfst/632 ; DOI :

https://doi.org/10.4000/rfst.632

\section{(c) (i) (2) (2)}

La Revue francophone sur la santé et les territoires est mise à disposition selon les termes de la Licence Creative Commons Attribution - Pas d'Utilisation Commerciale - Partage dans les Mêmes Conditions 4.0 International. 


\section{LES CIRCULATIONS EN SANTÉ : DES PRODUITS, DES SAVOIRS, DES PERSONNES} EN MOUVEMENT : ARGUMENTAIRE

Dans le prolongement du courant de pensée du «mobility turn », la mobilité est considérée comme un phénomène socio-spatial qui englobe les êtres (humains/non-humains, vivants/non vivants) en mouvement, les objets et techniques, les savoirs et les idées. Outre l'intérêt porté sur les flux et leurs composantes, les travaux sur les mobilités se sont également attachés à étudier les ancrages territoriaux de ces dernières dans les lieux de départ et d'arrivée ainsi que dans les espaces de transition (Scheller \& Urry 2006). Dans le cadre des soins, des contextes politiques, économiques, juridiques et sanitaires spécifiques font ainsi émerger des mobilités qui s'imposent parfois aux individus et façonnent leurs pratiques thérapeutiques. Des difficultés d'accès aux structures de soins, aux médicaments, aux ressources économiques constituent ainsi le terreau des mobilités sanitaires. Celles-ci sont en majorité internes à l'échelle des Etats-nations mais ce numéro thématique s'intéresse également aux mobilités transnationales qui impliquent le franchissement d'une ou de plusieurs frontières internationales.

L'objectif de ce dossier thématique est d'interroger la construction d'une catégorie émergente en sciences sociales que sont les circulations en santé dont la mondialisation et la notion de santé globale sont des éléments clés.

Dans le contexte général de la mobilité comme phénomène social, l'usage du terme de circulation apparaît intéressant à explorer pour mieux caractériser les mouvements de va-et-vient des individus entre différents contextes sanitaires ainsi que le pouvoir transformateur et thérapeutiques du mouvement (Kaspar et al. 2019). A travers les circulations en santé, il s'agira de réinterroger l'ambivalence entre l'uniformisation globale des marchés et l'ancrage local des pratiques (Appadurai 2001) en mesurant l'adéquation ou au contraire le décalage entre les injonctions globales en santé et les normes thérapeutiques locales.

Des géographes ont mis en évidence un certain penchant de la recherche sur les mobilités pour les déplacements réservés aux élites politiques, économiques ou sociales (ex : tourisme médical des pays des Nords vers les Suds) a contrario de déplacements plus ordinaires (Gatrell 2013). Ce dossier constitue un espace pour documenter ces circulations plus ordinaires dans le champ de la santé et mieux saisir la manière dont les corps, les connaissances médicales, les produits se transforment pendant et à l'issue des circulations.

De nombreux enjeux gravitent donc autour de cette notion de circulations en santé et les textes qui composent ce dossier traitent principalement des thématiques suivantes : (1) La circulation des savoirs médicaux et des produits en santé ; (2) La circulation des personnes, praticiens et patients ; (3) la gouvernance globale de la santé dans le contexte de la circulation accrue des risques sanitaires.

\section{Références :}

Appadurai Arjun, 2001. Après le colonialisme. Les conséquences culturelles de la globalisation, Paris, Payot, coll. "Petite Bibliothèque Payot", 333p.

Gatrell, Anthony C. 2013. "Therapeutic Mobilities: Walking and 'Steps' to Wellbeing and Health" Health \& Place, 22: 98-106.

Kaspar Heidi, Walton-Roberts Margaret, Bochaton Audrey, 2019. Therapeutic mobilities, Mobilities, 14 (1): 1-19.

Sheller Mimi and Urry John, 2006. The new mobilities paradigm. Environment and Planning A, 38(2): 207-226. 